\title{
A BESSEL FUNCTION EXPANSION
}

\section{MORRIS KLINE ${ }^{1}$}

1. Introduction. Some work in the theory of electromagnetic wave guides led to the following problem, namely, to obtain a useful approximation for the expression

$$
J_{\nu}(k R) N_{\nu}(k R+k a)-J_{\nu}(k R+k a) N_{\nu}(k R),
$$

wherein $J$, and $N_{\nu}$ are the Bessel functions of the first and second kind of order $\nu$, and under the conditions that $R$ be large and that $\nu=d R$, where $d$ is any real constant. It is perhaps worth while to record the result, particularly because it is obtained by rather elementary means. The result is a Taylor's expansion of the expression (1) about $R=\infty$ as follows:

$$
\begin{aligned}
& J_{\nu}(k R) N_{\nu}(k R+k a)-J_{\nu}(k R+k a) N_{\nu}(k R) \\
& =\frac{2}{\pi}\left[\frac{a}{R} \frac{\sin x}{x}+\frac{a^{2}}{2 R^{2}}\left\{\frac{\sin x}{\gamma x}-\left(1+\frac{1}{\gamma}\right) \cos x\right\}+\cdots\right]
\end{aligned}
$$

wherein $x^{2}=-k^{2} a^{2} \gamma, \gamma=\left(d^{2} / k^{2}\right)-1$ and $\nu=d R$. A similar result is also noted below for the expression:

$$
J_{\nu}^{\prime}(k R) N_{\nu}^{\prime}(k R+k a)-J_{\nu}^{\prime}(k R+k a) N_{\nu}^{\prime}(k R) .
$$

Physical interpretation of the two results is given in $\$ 3$.

It might be remarked that it did not appear possible to obtain any simple result with the usual asymptotic expansions for the individual Bessel functions.

2. The derivation of the basic expansion. Let $\alpha=k a$ and $\beta=k R$ in (1) and let us denote it by $G(\alpha, \beta)$. We shall, for the time being, regard the order $\nu$ of the Bessel functions $J_{\nu}$ and $N_{\nu}$ as fixed and expand $N_{\nu}(\beta+\alpha)$ and $J_{\nu}(\beta+\alpha)$ in powers of $\alpha$ at the point $\beta$. We obtain:

$$
\begin{aligned}
G(\alpha, \beta) & =J_{\nu}(\beta)\left\{N_{\nu}(\beta)+\alpha N_{\nu}^{\prime}(\beta)+\left(\alpha^{2} / 2\right) N_{\nu}^{\prime}(\beta)+\cdots\right\} \\
& -N_{\nu}(\beta)\left\{J_{\nu}(\beta)+\alpha J_{\nu}^{\prime}(\beta)+\left(\alpha^{2} / 2\right) J_{\nu}^{\prime \prime}(\beta)+\cdots\right\} .
\end{aligned}
$$

The functions $J_{\nu}(z)$ and $N_{\nu}(z)$ are analytic except at $z=\infty$ and, in the case of the second one, at $z=0$. The Taylor series representation is therefore valid for all values of $\alpha$ and $\beta$ chosen subject to these restric-

Received by the editors November 24, 1948 and, in revised form, April 27, 1949.

${ }^{1}$ Work on this paper was made possible by funds provided by the Watson Laboratories, Air Materiel Command, United States Air Force, under a contract with New York University. 
tions; the series must converge absolutely; and by an allowable rearrangement of its terms $G(\alpha, \beta)$ may be written as

$$
G(\alpha, \beta)=\sum_{n=1}^{\infty} \frac{\alpha^{n}}{n !} D_{0, n}(\beta)
$$

where we have adopted the notation, with respect to any variable $z$,

$$
D_{i, j}(z)=J_{\nu}^{[i]}(z) N_{\nu}^{[j]}(z)-J_{\nu}^{[i]}(z) N_{\nu}^{[i]}(z),
$$

the superscript $[i]$ meaning the $i$ th derivative with respect to $z$. We shall investigate the expression (5) with a view to ultimately finding those terms which may justifiably be regarded as the leading terms in $1 / R$ of the expansion (5) under the condition $\nu=d R .^{2}$

Let $u(z)$ and $v(z)$ denote any two independent solutions of the Bessel equation of order $\nu$. Then $u^{\prime \prime}=-z^{-1} u^{\prime}+z^{2}\left(\nu^{2}-z^{2}\right) u$, and $v^{\prime \prime}$ $=-z^{-1} v^{\prime}+z^{-2}\left(\nu^{2}-z^{2}\right) v$. Substituting these equations into $D_{2, r}$ gives immediately that $D_{2, r}=-z^{-1} D_{1, r}+z^{-2}\left(\nu^{2}-z^{2}\right) D_{0, r}$. If we set:

$$
\rho=z^{-1}, \quad \gamma=z^{-2}\left(\nu^{2}-z^{2}\right)=\nu^{2} \rho^{2}-1,
$$

we may write

$$
D_{2, r}=-\rho D_{1, r}+\gamma D_{0, r \cdot}
$$

It is immediate, from equation (6), that if we let the symbol $\delta$ stand for differentiation with respect to $z$ then:

$$
\delta D_{i, j}=D_{i+1, j}+D_{i, j+1} \text {. }
$$

By use of formulas (9) and (8) we readily establish that

$$
D_{0, n}=(2 \delta+\rho) D_{0, n-1}+\left(\gamma-\rho \delta-\delta^{2}\right) D_{0, n-2} \text {. }
$$

We introduce the notation $\Delta_{n}=(\pi / 2) D_{0, n}$, the factor $\pi / 2$ being chosen to cancel a $2 / \pi$ which will appear later in every $D_{0, n}$. Also we introduce the operators:

$$
\sigma=2 \delta+\rho \text { and } \tau=\gamma-\rho \delta-\delta^{2} .
$$

Then relation (10) above becomes the basic recursion formula:

$$
\Delta_{n}=\sigma \Delta_{n-1}+\tau \Delta_{n-2} \text {. }
$$

The formulas for $\Delta_{n}$ for the first few integers $n$ are well known, ${ }^{3}$ and

2 A special case of expression (5) for the case $>=0$ is used in Schelkunoff, S., The electromagnetic theory of coaxial transmission lines and cylindrical shields, Bell System Technical Journal vol. 13 (1934) p. $532 \mathrm{ff}$. See p. 562 in particular.

${ }^{3}$ For $n=0,1, \cdots, 5$, the function $D_{0, n}$ can be found in Watson, G. N., Introduction to the theory of Bessel functions, New York, Macmillan, 1945, p. 76. Formula (12) provides a convenient way of deriving as many more as may be needed. 
easily derived. The first two are $\Delta_{0}=0$ and $\Delta_{1}=\rho$. From the first two, and the recursion relation (12), we see that $\Delta_{2}=-\rho^{2}$, since $\rho$ is $z^{-1}$. In general, the expression $\Delta_{n}$ is a polynomial in $\rho$ and $\nu$ of increasing order in $\rho$ and $\nu$. It is also a polynomial in $\rho$ and $\gamma=\left(\nu^{2} \rho^{2}-1\right)$. The form of these polynomials becomes exceedingly complicated with increasing $n$.

We are interested in the dependence of $G(\alpha, \beta)$ on $1 / R$ as $R \rightarrow \infty$ and $\nu=d R$. To obtain this relationship we intend to let $1 / \rho=z=k R$, whereupon the combination $\nu \rho$, and therefore $\gamma$, will be of zero order in $1 / R$. The variable $\rho$ itself will be of first order in $1 / R$. Guided by these facts we shall seek out in each $\Delta_{n}$ the terms of total orders one and two in $1 / R$, discarding those of higher order.

We shall prove by induction that for all $n \geqq 1$

(a) $\Delta_{2 n-1} \sim \rho \gamma^{n-1}$

(b) $\Delta_{2 n} \sim-n(2 n-1) \rho^{2} \gamma^{n-1}-2 n(n-1) \rho^{2} \gamma^{n-2}$,

where the explicit terms on the right are exact, and where all terms omitted are of the form $P \rho^{o+2} \gamma^{m}, s>0, P$ a function of $n$. There are a finite number of such terms (for each $n$ ) and each of them, as remarked, is of at least third order in the ultimate variable $1 / R$.

By the definitions of $\sigma$ and $\tau$ it follows that for every $m \geqq 0$,

(i) $\quad \sigma\left\{\rho \gamma^{m}\right\}=-(1+4 m) \rho^{2} \gamma^{m}-4 m \rho^{2} \gamma^{m-1}$;

(ii) $\tau\left\{\rho \gamma^{m}\right\} \sim \rho \gamma^{m+1}$; (iii) $\sigma\left\{\rho^{2} \gamma^{m}\right\} \sim 0$;

(iv) $\tau\left\{\rho^{2} \gamma^{m}\right\} \sim \rho^{2} \gamma^{m+1}$;

(v) $\sigma\left\{\rho^{s+2} \gamma^{m}\right\} \sim 0$ and $\tau\left\{\rho^{s+2} \gamma^{m}\right\} \sim 0$, $s>0$;

the symbol $\sim$ being understood as directly above.

Turning now to formulas (13), note first that they are true in the special case $n=1$, and we must verify that if they hold for a given $n$ then they hold also for $n+1$. By the recursion formula (12), $\Delta_{2 n+1}$ $=\sigma \Delta_{2 n}+\tau \Delta_{2 n-1}$. Assuming that formula (13) holds for $n$, we obtain $\Delta_{2 n+1}=-n(2 n-1) \sigma\left\{\rho^{2} \gamma^{n-1}\right\}-2 n(n-1) \sigma\left\{\rho^{2} \gamma^{n-2}\right\}+\left\{\rho \gamma^{n-1}\right\}$. Therefore, by formulas (14), $\Delta_{2 n+1} \sim \rho \gamma^{n}$. Notice that in the case $n=1$, where the middle term involving $\boldsymbol{\gamma}^{n-2}$ might seem disquieting, this term is not actually present because of the factor $(n-1)$ in its coefficient. This proves formula (13a) for $\Delta_{2 n+1}$.

To prove formula (13b) for $\Delta_{2 n+2}$ we write formula (12) in the form $\Delta_{2 n+2}=\sigma \Delta_{2 n+1}+\tau \Delta_{2 n}$. Now we substitute for $\Delta_{2 n+1}$ from the preceding paragraph and for $\Delta_{2 n}$ from formula (13b). Then, $\Delta_{2 n+2}$ 
$\sim \sigma\left\{\rho \gamma^{n}\right\}+\tau\left\{-n(2 n-1) \rho^{2} \gamma^{n-1}-2 n(n-1) \rho^{2} \gamma^{n-2}\right\}$. Finally by formulas (14), $\Delta_{2 n+2} \sim-(n+1)(2 n+1) \rho^{2} \gamma^{2}-2(n+1) n \rho^{2} \gamma^{n-1}$. This concludes the demonstration of both formulas (13).

We now let $z=\beta$ (or $k R$ ) and substitute the approximations to $\Delta_{n}$ given by formulas (13) in the series (5), after replacing $D_{0, n}$ by $(2 / \pi) \Delta_{n}$. The use of approximations (13) in the terms of this series amounts to regarding the series as one in two variables $\alpha$ and $\beta$ and to a reordering of the terms. We postpone the justification for this step $^{4}$ and turn at once to the series.

First we separate $\sum_{n=1}^{\infty} \alpha^{n} / n ! \Delta_{n}$ into the sum of an "odd" and "even" series, $\sum_{m=1}^{\infty} \alpha^{2 m-1} /(2 m-1) ! \Delta_{2 m-1}$ and $\sum_{m=1}^{\infty} \alpha^{2 m} /(2 m) ! \Delta_{2 m}$. Then we substitute for each of the $\Delta^{\prime}$ 's the value from (13). Hence

$$
\begin{aligned}
\sum_{n=1}^{\infty} \frac{\alpha^{n}}{n !} \Delta_{n}= & \frac{1}{\beta} \sum_{m=1}^{\infty} \frac{\alpha^{2 m-1}}{(2 m-1) !} \gamma^{m-1} \\
& -\frac{1}{\beta^{2}} \sum_{m=1}^{\infty} \frac{\alpha^{2 m}}{(2 m) !}\left[m(2 m-1) \gamma^{m-1}+2 m(m-1) \gamma^{m-2}\right] \\
& + \text { remainder. }
\end{aligned}
$$

Each term of this remainder is of the form $\left(1 / \beta^{s}\right) f_{s}(\alpha, \gamma), s \geqq 3$, with $f_{s}$ an appropriate series in $\alpha$ whose coefficients are polynomials in $\gamma$.

We introduce $x^{2}=-\alpha^{2} \gamma$; as we shall see later, $x$ is real and positive in applications. By simple manipulations using, in the last series, the relation $n-1=(2 n-1) / 2-1 / 2$, we may express this as

$$
\begin{aligned}
\sum_{n=1}^{\infty} \frac{\alpha^{n}}{n !} \Delta_{n}= & \frac{\alpha}{x \beta} \sum_{m=1}^{\infty} \frac{(-1)^{m-1} x^{2 m-1}}{(2 m-1) !}-\frac{\alpha^{2}}{2 \beta^{2}} \sum_{m=1}^{\infty} \frac{(-1)^{m-1} x^{2 m-2}}{(2 m-2) !} \\
& +\frac{1}{2} \frac{\alpha^{2}}{\gamma x \beta^{2}} \sum_{m=1}^{\infty} \frac{(-1)^{m-1} x^{2 m-1}}{(2 m-1) !} \\
& -\frac{\alpha^{2}}{2 \gamma \beta^{2}} \sum_{m=1}^{\infty} \frac{(-1)^{m-1} x^{2 m-2}}{(2 m-2) !}+\text { remainder. }
\end{aligned}
$$

Substituting the trigonometric functions appropriate to the summations gives

$$
\begin{aligned}
G(\alpha, \beta)= & \frac{2}{\pi} \sum_{1}^{\infty} \frac{\alpha^{n}}{n !} \Delta_{n}=\frac{2 \alpha}{\beta \pi} \frac{\sin x}{x} \\
& +\frac{2 \alpha^{2}}{\pi \beta^{2}}\left[\frac{\sin x}{\gamma x}-\left(1+\frac{1}{\gamma}\right) \cos x\right]+\text { remainder. }
\end{aligned}
$$

${ }^{4}$ The justification is given in the appendix. The appendix also proves that the expression (2) is a Taylor's expansion about $R=\infty$. 
This is the result given in formula (2).

The notation in formula (6) and the formulas (12) and (13) are adequate to treat expression (3). If we again let $\alpha=k a$ and $\beta=k R$ and denote the expression by $\Gamma(\alpha, \beta)$, we obtain

$$
\begin{aligned}
\Gamma(\alpha, \beta)= & -\frac{2}{\pi} \frac{\alpha \gamma}{\beta} \frac{\sin x}{x} \\
& +\frac{\alpha^{2}}{\pi \beta^{2}}\left[(\gamma+1)\left(\frac{\sin x}{x}+\cos x\right)+\frac{\gamma \sin x}{x}\right] \\
& + \text { remainder. }
\end{aligned}
$$

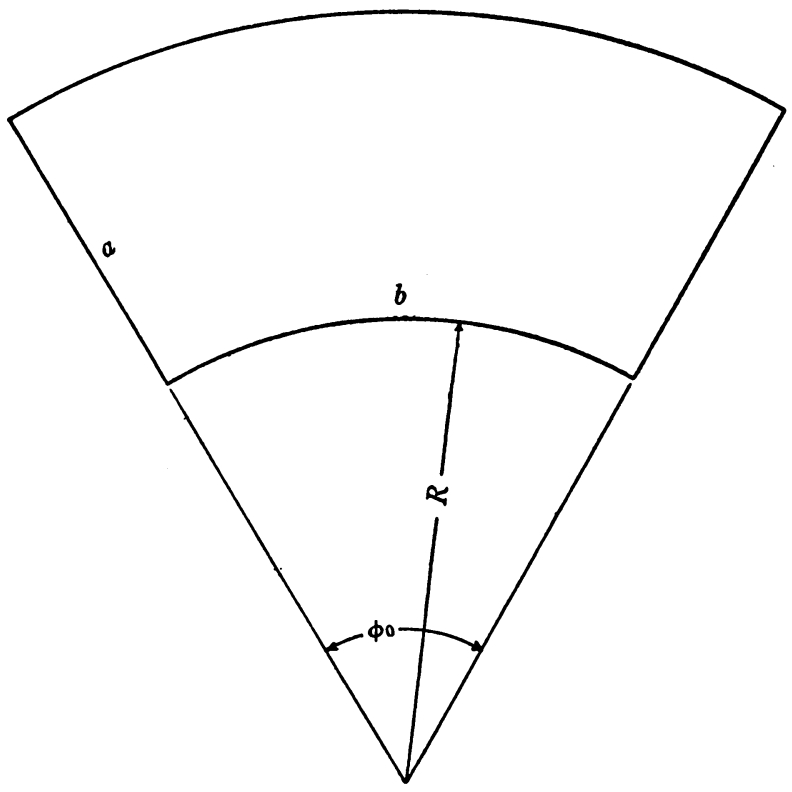

FIG. 1

3. Physical interpretation of the result. Given a sector of a coaxial guide, the cross-section of which is indicated in Fig. 1, the longitudinal component of the electrical field supported by such a guide is given by the expression ${ }^{5}$

$$
E_{z}=\left[K N_{\nu}(k R) J_{\nu}(k r)-K J_{\nu}(k R) N_{\nu}(k r)\right] \sin \nu \phi e^{j \omega t+h z}
$$

where $r, \phi$, and $z$ are the cylindrical coordinates employed, $\nu=R m \pi / b$, and $b=R \phi_{0}$. The quantity $K$ is determined by the amplitude of the

S. Ramo and J. R. Whinnery, Fields and waves in modern radio, New York, Wiley, 1944, pp. 325-326 and pp. 333-335. 
field excited in the guide. $k$ is determined by setting the left side of equation (2) equal to 0 . This equation yields an infinite number of values of $k$ for any one value of $\nu$, each value of $k$ thus determining one mode of the guide.

It is, incidentally, true that setting the right-hand side of equation (2) equal to zero gives an implicit equation for $k$ from which it is possible to determine $k$ to two terms. However, this value of $k$ may also be obtained in other ways. We shall merely note for later reference that if we do set this right-hand expression equal to 0 , multiply by $R$, and let $R$ become infinite, we obtain $\sin x=0$. Since $x=\left(-\alpha^{2} \gamma\right)^{1 / 2}=a\left(k^{2}-d^{2}\right)^{1 / 2}$, we must have $a\left(k^{2}-d^{2}\right)^{1 / 2}=n \pi, n=0$, $\pm 1, \pm 2$. However $d=m \pi / b$ and so $\left.k\right|_{R=\infty}=\left(n^{2} \pi^{2} / a^{2}+m^{2} \pi^{2} / b^{2}\right)^{1 / 2}$, which is the formula for the cutoff frequency of a rectangular waveguide supporting the $T M_{m, n}$ mode.

If one holds $a$ and $b$ fixed and allows $R$ to become infinite while $R \phi_{0}=b$, one sees geometrically that the coaxial sector becomes a rectangle with dimensions $a$ and $b$. The physical interpretation of the basic result (2) is made in terms of this rectangle as follows. As $R$ becomes infinite the factor $\sin \nu \phi=\sin R m \pi \phi / b$ in expression (17) approaches $\sin m \pi y / b$, where $y=R \phi$. The limiting value of the factor involving the Bessel functions is given by the result of this paper; for, by letting $t=r-R$ this factor becomes, by virtue of formula (2),

$$
\begin{gathered}
\frac{2 K}{\pi} \frac{t}{R} \frac{\sin x}{x}+\frac{K}{\pi} \frac{t^{2}}{R^{2}}\left\{\frac{\sin x}{\gamma x}-\left(1+\frac{1}{\gamma} \cos x\right)\right\} \\
+ \text { terms of higher order in } \frac{1}{R},
\end{gathered}
$$

where $x^{2}$ is now $-k^{2} t^{2} \gamma$ and $\gamma=\left(d^{2} / k^{2}\right)-1$. To determine the limit of this expression it is necessary to normalize it, for the field strength should not be dependent upon $R$. Normalization may be accomplished by requiring the amplitude of the factor (18) at $r=R+a / 2$ to be 1 for each value of $R$. Then $1 / K=N_{\nu}(k R) J_{\nu}(k R+k a / 2)$ $-J_{\nu}(k R) N_{\nu}(k R+k a / 2)$. Again by formula (2) of this paper, wherein we now let $a$ be $a / 2$, and by simple reductions and the taking of a limit as $R$ becomes infinite, we find that expression (18) has the limiting form $L \sin \left(k^{2}-m^{2} \pi^{2} / b^{2}\right)^{1 / 2}(r-R)$, where $L$ is independent of $R$ and $t$. The $k$ here is $\left.k\right|_{R=\infty}$ which we have seen has the value $n^{2} \pi^{2} / a^{2}+m^{2} \pi^{2} / b^{2}$. This result shows that expression (18) permits us to exhibit the field in a coaxial sector as a function which approaches the field in the rectangular guide approached by the sector as $R$ becomes infinite. 


\section{Appendix}

This appendix will supply several theoretical details needed to complete the mathematics of the paper. In particular, it will justify the rearrangement of terms made in \$2. This rearrangement converted a power series in $\alpha$, with coefficients dependent upon $\nu$ and $\rho$, into a power series in $\rho$ with coefficients dependent on $k$ and $d$ and subject to the condition that $\nu$ is a constant over $\rho$. As the body of the paper shows, when $\nu$ and $\rho$ are replaced by their values in terms of $R$, the combination $\nu \rho$ is independent of $R$, the latter being the essential variable for the purpose of this paper.

Actual calculation of the quantities $\Delta_{2}, \Delta_{3}, \Delta_{4}, \cdots$, by operating with the basic recursion formula (12) of the paper, indicates that $\Delta_{n}$ for even $n$ can be expressed as a polynomial in even powers of $\rho$ with coefficients which are polynomials in even power of $\nu$. Likewise, for odd $n, \Delta_{n}$ can be expressed as a polynomial in odd powers of $\rho$ with coefficients which are polynomials in even powers of $\nu$. More formally stated, we have the following theorem.

Theorem A.

$$
\Delta_{2 n-1}(\rho)=\sum_{i=0}^{n-1} P_{i}^{2 n-1} \rho^{2 n-2 i-1} \text { wherein } P_{i}^{2 n-1}=\sum_{j=0}^{n-1-i} a_{i, j}^{2 n-1} \nu^{2(n-1-i-j)}
$$

and

$$
\Delta_{2 n}(\rho)=\sum_{i=0}^{n-1} P_{i \rho}^{2 n} \rho^{2 n-2 i} \quad \text { wherein } \quad P_{i}^{2 n}=\sum_{j=0}^{n-1-i} a_{i, j}^{2 n} \nu^{2(n-1-i-j)} .
$$

Moreover, the coefficients $a_{\mathfrak{i}, ~}^{n}$ satisfy the relations

(a)

$$
\begin{aligned}
a_{i, j}^{2 n+1}= & -(4 n-4 i-1) a_{i, j-1}^{2 n} \\
& +a_{i, j}^{2 n-1}-(2 n-2 i-1)^{2} a_{i, j-1}^{2 n-1}-a_{i-1, j}^{2 n-1}, \\
a_{i, j}^{2 n+2}= & -(4 n-4 i+1) a_{i, j}^{2 n+1} \\
& +a_{i, j}^{2 n}-(2 n-2 i-2)^{2} a_{i, j-1}^{2 n}-a_{i-1, j}^{2 n},
\end{aligned}
$$

provided that $a_{\mathfrak{i}, j}^{m}=0$ when $i+j>(m-1) / 2$, or $i<0$, or $j<0$, or $m<1$.

The proof of this theorem is readily made by mathematical induction utilizing the recursion formula (12).

It is now necessary to investigate the pattern of signs of the coeffcients of the polynomials $P_{\boldsymbol{r}}^{m}$ with a view to showing that we are dealing with an absolutely convergent series. We next prove the following theorem. 
Theorem B. (1) The sign of $a_{i, j}^{m}=(-1)^{m+i+1}, m=1,2,3, \cdots$; $0 \leqq i \leqq(m-1) / 2$.

(2) The sign of $a_{i, j-1}^{2 n-1}+(2 n-2 i-2) a_{i, j-2}^{2 n-2}=(-1)^{i}, i+j \leqq n-1$.

(3) The sign of $a_{i, j-1}^{2 n}+(2 n-2 i-1) a_{i, j-1}^{2 n-1}=(-1)^{i+1}, i+j \leqq n-1$.

Proof. It is (1) only, of Theorem B, which will be needed ultimately; however, the nature of the proof forces us to be concerned with parts (2) and (3). The proof is by mathematical induction. By inspection of $\Delta_{2 n-2}, \Delta_{2 n-1}$, and $\Delta_{2 n}$, when $n=1$ we find all three statements to be true. We now assume these statements true for $2 n, 2 n-1$, and $2 n-2$ and proceed to the main step of the inductive argument.

It follows from relation (a) of Theorem A, with $j$ replaced by $j-1$ and the terms regrouped, that for all $i, j$

$$
\begin{aligned}
a_{i, j-1}^{2 n+1}+(2 n-2 i) a_{i, j-2}^{2 n} \\
=-(2 n-2 i-1)\left\{a_{i, j-2}^{2 n}+(2 n-2 i-1) a_{i, j-2}^{2 n-1}\right\} \\
\quad+a_{i, j-1}^{2 n-1}-a_{i-1, j-1}^{2 n-1} .
\end{aligned}
$$

By the induction assumption (3) the sign of the first term on the right is $(-1)^{i+2}=(-1)^{i}$; the sign of the second term, by assumption (1), is $(-1)^{2 n-1+i+1}=(-1)^{i}$; and the sign of the third term is $(-1)^{1+(2 n-1)+(i-1)+1}=(-1)^{i}$. It follows that assumption (2) holds for $n+1$.

By the induction assumption (1) the sign of $a_{i, j-2}^{2 n}$ is $(-1)^{i+1}$ and the sign of the entire left side of $(a)$ is $(-1)^{i}$ by the previous paragraph. It follows therefore that the term $a_{i, j-1}^{2 n+1}$ on the left side of (a) has the sign $(-1)^{i}$. This step completes the inductive proof of statement (1) for the case where $m=2 n+1$.

To establish statement (3) of the theorem we use relation (b) of Theorem A. If in that relation we replace $j$ by $j-1$ and rearrange terms slightly, we obtain

$$
\begin{aligned}
a_{i, j-1}^{2 n+2}+ & (2 n-2 i+1) a_{i, j-1}^{2 n+1} \\
= & -(2 n-2 i) a_{i, j-1}^{2 n+1}-(2 n-2 i-2) a_{i, j-2}^{2 n}+a_{i, j-1}^{2 n}-a_{i-1, j-1}^{2 n} \\
= & -(2 n-2 i)\left\{a_{i, j-1}^{2 n+1}+(2 n-2 i) a_{i, j-2}^{2 n}\right\} \\
& +2(4 n-4 i-2) a_{i, j-2}^{2 n}+a_{i, j-1}^{2 n}-a_{i-1, j-1}^{2 n} .
\end{aligned}
$$

Since we have already established relation (2) for $n+1$, it follows that the sign of the first term on the right is $(-1)^{i+1}$. This is also the sign of each of the remaining terms in view of the inductive assumption for relation (1). Hence the right side and therefore the left side has the sign $(-1)^{i+1}$, which establishes relation (3) for $n+1$. At the 
same time, since $a_{i, j-1}^{2 n+1}$ has the sign $(-1)^{i}$ by the inductive proof of relation (1) when $m$ is odd, it must be that the sign of $a_{i, j-1}^{2 n+2}$ is $(-1)^{i+1}$ and this is the proof of relation (1) for $m=2 n+2$, which completes the entire proof of that relation.

In consequence of (1) of Theorem $B$, the coefficients $a_{\mathfrak{i}, j}^{m}$ in the polynomials $P_{i}^{m}$ in Theorem A are all of one sign, depending on $m$ and $i$, and we obtain the following corollary.

COROLlaRy I.

$$
\begin{aligned}
& (-1)^{i} P_{i}^{2 n-1}=\sum_{j=1}^{n-i-1}\left|a_{i, j}^{2 n-1}\right| \nu^{2(n-1-i-j)}, \\
& (-1)^{i+1} P_{i}^{2 n}=\sum_{j=1}^{n+i=1}\left|a_{i, j}^{2 n}\right| \nu^{2(n-1-i-j)} .
\end{aligned}
$$

We now let $\rho=(-1)^{1 / 2} r$ and substitute this and the results of Corollary I into the expressions for $\Delta_{2 n-1}$ and $\Delta_{2 n}$ of Theorem A. Further we let $\alpha=(-1)^{1 / 2} A$. Then we have the following corollary.

Corollary II. For $\alpha=(-1)^{1 / 2} A$ and $\rho=(-1)^{1 / 2} r, \quad-\alpha^{m} \Delta_{m}(\rho)$ $=A^{m} Q_{m}(r, \nu), m=1,2,3, \cdots$, where the coefficients in the polynomial $Q_{m}$ are all positive.

We are now near the conclusion. We recall that the Bessel functions $J_{\nu}(z)$ and $N_{\nu}(z)$ are analytic functions of the argument $z$ everywhere except at $z=\infty$, and for the second of these functions at $z=0$. It follows at once that $G(\alpha, \beta)=J_{\nu}(\beta) N_{\nu}(\alpha+\beta)-J_{\nu}(\alpha+\beta) N_{\nu}(\beta)$ is analytic in $\alpha$ and $\beta$ excepting possibly at the loci $\alpha=0, \alpha=\infty, \beta=0, \beta=\infty, \alpha+\beta=0$.

We already know from formula (5) that $(2 \pi) G\left(\alpha, \rho^{-1}\right)$ $=\sum_{m=1}^{\infty}\left(\alpha^{m} / m !\right) \Delta_{m}(\nu, \rho)$. The series on the right converges for all $\alpha$ and $\rho=\beta^{-1}$ which are not on the exceptional loci. By Corollary II, $(-2 / \pi) G\left((-1)^{1 / 2} A, 1 /(-1)^{1 / 2} r\right)=\sum_{m=1}^{\infty}\left(A^{m} / m !\right) Q_{m}(\nu, r)$. This power series in $A$ converges for every $A$, for every fixed $r$, with exceptions as above, provided, of course, that in the summation we regard the terms in each $Q_{m}(\nu, r)$ as locked in a parenthesis, the entire polynomial serving as coefficient for $A^{m}$. But now, since each coefficient of $Q_{m}(\nu, r)$ is positive, we may regard the series on the right as a simple sequence of terms ( $A$ and $r$ being thought of as fixed, arbitrary, real positive numbers) and we may certainly rearrange the terms of this simple sequence in any order, and reintroduce parentheses in any way we wish. The resulting series will converge to the same value as the original series. ${ }^{6}$ Moreover $r$ and $A$ may also assume imaginary values

${ }^{6}$ See, for example, T. J. Bromwich, An introduction to the theory of infinite series, London, Macmillan, 1908, articles 31 and 33. 
and the rearrangement of terms remains justified, for the absolute values of the imaginary $r$ and $A$ are the same as for real $r$ and $A$ and the convergence of the absolute values suffices to justify rearrangement.

An entirely analogous argument disposes of the same problem for the derivation of formula (16).

It should now be noted that the rearranged series, that is, formula (2), is a power series in $1 / R$ which, because it equals the original series for $G(\alpha, \beta)$, certainly converges for all values of $R$ except, perhaps, the few exceptional ones $R=0, R=\infty$, and $R=-a$. As a power series in $1 / R$ it must also converge then for $R=\infty$ and it represents the function $G(\alpha, \beta)$ at $R=\infty$ if we define the function to have value 0 at that point. Formula (15) is therefore a Taylor's expansion of $G(\alpha, \beta)$ around $R=\infty$ under the condition $\nu=m \pi R / b{ }^{7}$

NEW YORK UNIVERSITY

${ }^{7}$ Acknowledgment is hereby made of assistance from Dr. Leo Zippin in carrying out several inductive proofs of the paper. 